\title{
De quelques routines phraséologiques liées aux verbes parenthétiques dans les genres scientifiques
}

\author{
Francis Grossmann \\ Lidilem, E. A. 609, Univ. Grenoble \\ francis.grossmann@u-grenoble3.fr
}

\section{Introduction}

Existe-t-il des moyens linguistiques privilégiés, des routines phraséologiques spécialisées dans l'énonciation du constat scientifique ? Dans les travaux précédents menés à partir de la base Scientext (pour une synthèse récente, voir Tutin et Grossmann, 2013) nous avons pu identifier, pour le français, certaines des routines propres à l'écrit scientifique : elles ont des formats et des fonctions variés, et peuvent signaler la divergence ou démarcation, l'inscription dans une filiation scientifique, l'expression du consensus, etc. Ces routines s'inscrivent dans des configurations discursives plus larges, qui leur donnent tout leur sens : ainsi, le marquage d'un accord partiel précède-t-il fréquemment l'expression d'une divergence, elle-même s'inscrivant dans une séquence mettant en évidence l'originalité de la direction de recherche suivie par le chercheur. Le repérage des motifs du constat dans les genres scientifiques représente un enjeu particulier, tant pour la description linguistique des genres que pour la recherche d'informations, puisque l'on peut penser qu'à travers ce type de formules, sont mis en évidence les éléments accompagnant ou concluant le processus d'administration de la preuve ${ }^{1}$. Une remarque doit cependant inciter à la prudence : la forme prototypique du constat - scientifique ou pas - est l'assertion telle qu'elle s'exprime dans la phrase simple et elle ne comporte donc pas de marque particulière ; l'utilisation de formes spécifiques, telles que des verbes à la première personne (nous voyons que, nous constatons que ...) correspond à des fonctions pragmatiques qui visent souvent à impliquer le lecteur dans le co-constat, ou bien à rappeler des éléments déjà rencontrés. Cette fonction d'implication et de balisage textuel s'exprime notamment grâce à des incises et autres structures en comme (comme nous l'avons vu précédemment...), qui sont aussi des marqueurs de présupposition et ont souvent également un statut méta-énonciatif (voir Grossmann et Tutin, 2010a et 2010b). Le but principal de notre contribution est donc de tenter de faire la part, si la chose est possible, entre la rhétorique du balisage textuel (impliquant souvent un mécanisme présuppositionnel) et celle qui conduit à mettre en scène les constats scientifiques considérés comme essentiels par le chercheur. Est-il possible alors d'identifier les formules parenthétiques comme relevant essentiellement du premier dispositif? Et peut-on démêler, ne serait-ce que partiellement, ces deux niveaux de l'argumentation scientifique ? Avant de tenter de répondre à ces questions, il faut rappeler au préalable quelques éléments liés à l'énonciation scientifique.

Les voix énonciatives dans l'écrit scientifique sont principalement associables à nous et à on, marqueurs éminemment ambigus (Loffler-Laurian, 1980 ; Fløttum et al. 2006 ; Fløttum et al. 2007 ; Tutin 2010) qui peuvent renvoyer à la condition d'être humain, à l'auteur (singulier dans le nous ou on de modestie ou pluriel), à la communauté sociale ou à un sous-ensemble de celle-ci, exclusivement au lecteur dans certains emplois spécifiques, ou à des emplois inclusifs plus génériques associant à la fois l'auteur et la communauté de discours. La prise en charge de l'assertion pose des problèmes complexes dans l'écrit scientifique, étant donné la double contrainte suivant laquelle l'énonciateur doit se présenter à la fois comme le représentant d'une vérité universelle, échappant à la subjectivité, et en même temps comme proposant un point de vue d'auteur, apportant des éléments de connaissance nouveaux à l'édifice scientifique. Pour répondre à la première exigence, l'assertion revêt fréquemment la forme d'un constat généralisant, au présent de l'indicatif, évacuant fréquemment ces marqueurs de subjectivité que sont les 
pronoms personnels et ne comportant pas de verbe d'opinion, d'observation et de constat à la première personne :

1) Cette prédisposition aux cancers cutanés se manifeste précocement en moyenne à l'âge de 8 ans chez les patients XP contre 50-60 ans dans la population. [bio-the-284-body]

La phrase déclarative ne peut cependant être utilisée en toute légitimité que si, à un moment ou à un autre, est précisée la source des informations autorisant la généralisation, soit qu'il s'agisse de données présentées par l'auteur, soit que ce dernier s'appuie sur la littérature. C'est ce qui explique le mouvement dialogique qui conduit à associer le lecteur à l'examen des preuves empiriques, à travers une procédure de co-constat. Les verbes d'observation et de constat sont soit des verbes de perception (voir, observer) soit des verbes liés à la trace (noter) mais il s'agit bien, la plupart du temps, de mettre en scène une opération intellectuelle d'analyse de données plus que de simple perception ou d'activité graphique. Delplanque (2006) souligne le fait que les verbes de constat, tout comme les verbes de jugement (je trouve que...) sont explicitement construits à partir d'un sujet énonciateur renvoyant à un être du monde réel qui assume l'observation. Cette caractéristique les oppose aux verbes d'apparence dans lesquels " l'énonciateur évacue sa place de "constructeur », quitte à réapparaître, dans certains cas et dans certaines conditions, comme « récepteur » du procès »: Pierre m'a l'air triste. Les verbes de constat, quant à eux, sont dans les genres scientifiques très directement liés au dialogue intersubjectif qu'établit l'énonciateur.

\section{Corpus et objectifs}

Nous fondons notre analyse sur une exploitation de la partie française du corpus SCIENTEXT, tous genres (à l'exception des expertises) et toutes disciplines confondues, ce qui représente environ 4,8 millions de mots. Nous ne nous livrerons pas ici à une analyse statistique détaillée des formes les plus représentées dans le corpus, notre objectif principal étant plutôt de proposer une typologie des différentes routines de l'observation et du constat, permettant de poursuivre la description de ces structures, déjà esquissée précédemment (Grossmann, 2014). Nous appelons conventionnellement verbes d'observation et de constat (désormais VOC) les verbes synonymes d'observer et de constater qui sont utilisés comme support principal des routines étudiées. Si l'on choisit comme entrée le verbe constater, sans doute le plus prototypique, l'examen du Trésor de la langue française informatisé rend compte de deux emplois principaux, une fois mise de côté l'acception juridique («attester par un acte officiel ») : dans l'emploi imperfectif, constater, c'est « établir après examen l'existence ou l'authenticité d'un fait. » Dans l'activité scientifique, c'est plus particulièrement « s'assurer, au moyen d'observations scientifiques, de la réalité d'un fait ». En emploi perfectif, il peut s'agir de "prendre connaissance de quelque chose », ou, plus directement intéressant pour nous, de « se rendre compte de l'existence d'un fait ». La polysémie est donc assez réduite pour ce verbe. En revanche, son fonctionnement est assez spécifique dans l'écrit scientifique: dans un sens " textuel ", le constat énoncé correspond à des points (faits, idées, hypothèses...) considérés comme acquis par un développement antérieur, ou annoncés comme allant être démontrés dans la suite du texte. Les VOC ont en commun, dans les genres scientifiques, de servir de support à une assertion scientifique, généralement étayée par des éléments auxquels le lecteur du texte peut se référer. Globalement, on trouve effectivement un grand nombre de points communs entre eux, mais aussi des différences dans leur fonctionnement et leur utilisation. Parmi les points communs, il y a le fait que tous les verbes étudiés - à l'exception de s'apercevoir qui utilise presque exclusivement la construction en QUE_P - construisent leur objet en autorisant l'alternance Que_P et SN objet. La fréquence de la structure en QUE_P, et le rôle de modalisation épistémique que jouent ces verbes dans l'énoncé les rapprochent des verbes parfois appelés, plutôt improprement comme le note Borillo (1982), verbes « assertifs ». Les verbes « assertifs » (tels savoir, regretter, conclure, déduire, etc.) ont la propriété de présenter la proposition qu'ils introduisent comme vraie. Deuxième caractéristique importante : ils peuvent entrer dans des structures parenthétiques avec ou sans reprise anaphorique qui permettent alors de nuancer «cette prétention à la vérité » (Tuchais, 2012, p.157), et jouent alors le rôle d'adverbes d'énonciation. 
La liste des verbes étudiés est la suivante : voir ; remarquer, noter, observer ; s'apercevoir. Nous n'avons pas inclus parmi nos VOC, les verbes «de preuve» qui présupposent un constat : vérifier, prouver, démontrer ou encore établir. Ces derniers, sémantiquement proches des verbes de constat, mériteraient une étude spécifique. Nous avons sélectionné les emplois correspondant au sens de constat, en éliminant manuellement les énoncés dans lesquels ces verbes prennent un autre sens. En résumé, pour chaque verbe, nous avons considéré : la forme «simple»; la forme modalisée avec pouvoir, parce qu'elle est fréquemment associée à ces verbes; la forme avec nous; la forme avec on. Nous avons également examiné les différentes constructions syntaxiques. Nous présentons ici les traits saillants de cette étude 2 .

Parmi les verbes étudiés, deux se détachent nettement, par leur fréquence comme par leur centralité : ce sont observer et constater, le premier (471 occurrences sur un total de 1612) est, en raison de son sémantisme, davantage mobilisé pour décrire la phase empirique de la recherche, le second (409 occurrences), plus abstrait, pour en traduire les résultats. Ils sont suivis de voir (268 occ.), remarquer $(221$ occ.) et noter $(221$ occ.) enfin s'apercevoir dont nous avons trouvé seulement 23 occurrences pertinentes. Dans nombre de contextes, constater et observer sont interchangeables, observer pouvant prendre comme constater les valeurs imperfectives ou perfectives. Constater est plus fréquemment associé au nous académique qu'observer : un partage des rôles énonciatifs se dessine donc entre d'un côté nous constatons que, forme prototypique du constat conclusif de l'auteur et on observe $(+$ local.), construction privilégiée du co-constat incluant le lecteur.

Noter et remarquer semblent sémantiquement assez proches, en ce qu'ils établissent un constat incident, ayant parfois valeur d'ajout, de commentaire ou d'illustration à une affirmation qui précède. Remarquer comme noter sont fréquemment associés à des adverbiaux du type d'ailleurs, pourtant, néanmoins, aussi, ce qui semble indiquer qu'ils ont fondamentalement un rôle argumentatif et marquent soit un contreargument soit un ajout renforçant l'argument qui précède. Etant donné son emploi plus fréquent à la première personne, remarquer semble d'un emploi plus monologique que noter, qui a plus d'affinités avec le co-constat impliquant le lecteur, bien que là encore il s'agisse de simples tendances, les deux emplois étant toujours possibles.

Voir et s'apercevoir ont en commun de se situer plutôt sur le versant interprétatif du constat. Voir cependant a une très large palette d'utilisation, ce qui nous obligera à l'examiner à part : certains de ses emplois le conduisent à sortir du rôle de constatif pour revêtir une valeur évaluative (cf. on voit bien...) et un tri plus sévère que celui que nous avons réalisé se révèlerait nécessaire ; s'apercevoir, peu fréquent, est exclusivement utilisé pour marquer l'issue d'un processus d'observation.

Pour l'ensemble des verbes (sauf constater pour lequel le nombre d'emplois avec pouvoir est équivalent), se confirme le fait que la modalisation avec pouvoir est plus facilement utilisée avec on qu'avec nous, ce qui semble montrer ses affinités avec le co-constat.

\section{Les structures parenthétiques et le fonctionnement des incises}

\subsection{Les verbes parenthétiques et la conception dérivationniste}

Dans le discours, les structures parenthétiques du type je suppose peuvent jouer un rôle de modalisation épistémique, d'atténuateurs ou revêtent une valeur évidentielle. Le point important réside dans le fait que, même lorsque le verbe est enchâssant, il n'assure pas la prédication principale de l'énoncé. Ce fonctionnement implique donc fréquemment un décrochement énonciatif, du type de celui qu'effectuent également certains verbes de dire ou de pensée utilisés dans le cadre du discours rapporté. Il est naturel que ces verbes aient particulièrement intéressé les spécialistes de l'oral, dans la mesure où ils y sont extrêmement fréquents et qu'on observe des phénomènes d'ellipse ; dans la mesure aussi où la prosodie peut permettre de préciser les différences que peut impliquer, par exemple, la position de l'incise ou signaler le degré de pragmaticalisation. On retrouve cependant à l'écrit les principaux emplois repérés à l'oral, à l'exception de l'emploi responsif, illustré par l'exemple (2) : 
2) C'est dommage - Oui, je trouve.

Se pose entre autres la question de leur statut et des critères de leur définition. Généralement, la littérature prend pour acquis, à la suite d'Urmson (1952) que les verbes dits «parenthétiques » peuvent apparaître dans les trois positions : en initiale, en fin, et inséré au sein de l'énoncé. La discussion porte en particulier sur le caractère régissant du verbe. Ainsi, selon Gachet et Avanzi (2011), au plan syntaxique, il s'agit notamment de savoir si en position initiale, ces verbes régissent, ne régissent pas, ou régissent «faiblement» la complétive qui les suit. La fonction syntaxique de ce type de verbes lorsqu'ils sont en position d'incise est également fréquemment interrogée.

Cependant, on peut douter de l'existence même de cette catégorie, si on la construit sur cette seule base distributionnelle et - implicitement ou explicitement - transformationnelle. Certains auteurs, notamment de Cornulier (1978, p. 55 et $s q$ ) contestent l'intérêt même de construire une classe de verbes parenthétiques, au moins pour le français. De Cornulier discute en particulier la dérivation transformationnelle des incises, censée permettre le rétablissement d'une structure complétive. Il rappelle que l'incise n'est pas toujours un régisseur possible, comme le montrent bien certains exemples :

3) Encore une faute d'orthographe, me semble-il. - ? Il me semble qu'encore une faute d'orthographe.

Ces considérations concernent entre autres les verbes impliquant un dégagement de la responsabilité du locuteur :

4) D'autres propositions, plus adéquates croyons-nous, vous seront soumises sous peu.

De Cornulier (ouvr. cit. p. 59) signale que l'incise croyons-nous (ici exemple 4), affecte sémantiquement le caractère "plus adéquat» des propositions, non le fait qu'elles «seraient soumises sous peu ». Or l'expression plus adéquate n'est pas capable à elle seule de former une complétive : c'est la construction appositive dans sa relation avec les sujets « d'autres propositions » qui permet d'assurer la prédication sur laquelle porte l'incise. Sa conclusion générale débouche sur l'idée que les verbes dits parenthétiques ne le sont qu'en fonction de conditions particulières qui prennent en compte la négation, l'aspect, les verbes qui les régissent, les modifieurs adverbiaux, la nature du sujet, etc. Pour d'autres raisons, Recanati (1984) récuse également l'hypothèse transformationnelle et dérivationniste. Il rappelle la double lecture des verbes « asymétriques » tels que je crois que $p$, assymétrie qui existerait entre emploi descriptif et emploi non descriptif : en disant je crois que $p$, je peux en effet soit décrire mes croyances présentes soit asserter prudemment que $p$, ce qui correspond d'après lui à l'emploi non descriptif, assymétrique. Il considère comme construits normalement, avec une proposition principale et une proposition complétive, les énoncés descriptifs. En revanche, les incises du type je suppose, je crois, je te prie, je t'assure, etc. sont considérées comme des incises pragmatiques, à valeur adverbiale : «le rejet en incise de la proposition principale souligne son caractère d'appendice quasi métatextuel et met en valeur le fait que la complétive est le vrai énoncé» (ouvr. cit. p. 329). Le caractère assez figé de ces incises explique qu'on puisse les considérer comme faisant partie de la langue, et pouvant être consignées dans des dictionnaires. Pour toutes ces raisons, nous ne nous situerons pas dans une optique dérivationnelle, qui considèrerait comme acquise la possibilité d'une transformation complétive, ce qui à l'évidence complique inutilement l'analyse et se montre dans bien des cas contre-intuitif. L'approche non dériviationniste plaide en faveur d'une distinction claire entre les structures dans lesquelles les verbes assertifs introduisent une proposition complétive ou infinitive et les structures parenthétiques. Cela nous conduit donc à penser que ces dernières, même lorsqu'elles comportent un verbe constatif, n'ont qu'un rôle de modalisation, et n'expriment pas le constat en tant que tel. Voyons ce qu'il en est dans les genres scientifiques.

\subsection{Les structures parenthétiques dans l'écrit scientifique}

Une différence essentielle distingue les structures que nous avons repérées dans l'écrit scientifique de celles qui sont généralement étudiées par la littérature : le tour à la première personne est exclu en incise, sauf dans les incises progressives et des structures en comme (voir ci-après). Une caractéristique typique 
de l'écrit scientifique par rapport à l'emploi à l'oral est le recours à l'indéfini on, dont on sait qu'il peut avoir différentes valeurs, notamment dans l'écrit scientifique (on auteur, on auteur + lecteur, on communauté de discours, $c f$. Fløttum et al., 2007). De la même façon, les formes impersonnelles du type semble-t-il peuvent-elles être également incluses dans cette catégorie, bien que n'entrant pas dans la définition de verbes «à la première personne », dans la mesure où elles impliquent bien un point de vue de l'énonciateur.

Il nous semble donc judicieux de partir d'une définition nettement énonciative en approfondissant la piste du «décrochage» déjà formulée par Urmson, et en considérant le critère syntaxique lié aux trois positions comme secondaire, applicable dans certains cas mais pas dans tous. Les verbes parenthétiques sont donc pour nous essentiellement des verbes pouvant introduire un prédicat secondaire permettant une modalisation de l'assertion principale. La portée du commentaire énonciatif, comme le montre Wilmet (1998, p.177), permet aussi, plus clairement qu'à partir de critères purement morpho-syntaxiques, de distinguer incises et incidentes: tandis que «l'incise insère à $\mathrm{P}$ une sous-phrase $\Delta$ en guise de complément circonstanciel de l'énonciation », l'incidente «insère à la phrase P1 d'accueil une phrase $\mathrm{P} 2$ ». Ainsi, des séquences telles que semble-t-il ou il est vrai peuvent-elles être analysées comme des incises, tandis qu'une phrase intercalée, telle que à Dieu ne plaise! insérée après si j'avais dans la phrase Si j'avais vingt ans sera décrite comme une incidente. Dans le cas d'une incise telle que 5 :

5) Occupée par des Grecs depuis sa fondation, semble-t-il deux siècles plus tôt, Byzance est irrémédiablement devenue l'une des cibles du roi perse.

le commentaire énonciatif porte sur un prédicat spécifique (la fondation de Bysance) et non sur toute la phrase.

\section{Présentation des routines de l'observation et du constat}

\subsection{Les routines liées aux structures parenthétiques}

Une première famille de routines se définit par la conjonction de deux caractéristiques linguistiques : l'usage d'un VOC et le recours à une structure parenthétique, de type incise ou incidente. La question des incises, incidentes et autres structures méta-énonciatives a donné lieu à une littérature abondante. Comme explicité en 3.2., nous suivons Wilmet (1998 : 574) en considérant que le critère énonciatif représente un élément décisif pour définir les différents phénomènes regroupés sous les termes d'incises ou d'incidentes. Nous considèrerons également, comme Beyssade (2012, p.123-124) que les commentaires méta-énonciatifs du type

6) Paul, c'est malheureux, ne pourra pas venir

sont des propositions incidentes, dans lesquelles, au plan syntaxique, l'inversion du sujet n'est pas obligatoire, et qui sont autonomes sémantiquement, par rapport à la phrase hôte (elle peut être niée). En ce qui concerne les incises, elles sont définies, à la suite de de Cornulier, comme ne disposant pas de cette autonomie sémantique et syntaxique : elles sont fréquemment soumises à l'inversion du sujet (dit-il, paraît-il, semble-t-il), et ne peuvent être niées. De Cornulier comme Beyssade distinguent deux types d'incises: des incises «de discours", liées au discours rapporté, dans lesquelles l'inversion est obligatoire, et des incises "progressives ", à fonction épistémique ou dialogique comme je crois, tu vois ou vois-tu, qui se construisent ou non avec inversion et dont le sujet est toujours un pronom personnel ou impersonnel. Dans notre corpus, deux structures sont prototypiques : a) les structures comportant un pronom anaphorique objet du type on le voit; il s'agit d'incises, puisqu'elles ne disposent ni d'autonomie sémantique ni d'autonomie syntaxique; b) les structures en comme du type comme on le voit, qui comportent également un anaphorique, très proches des précédentes, sauf qu'il ne s'agit pas d'incises à proprement dit: elles introduisent majoritairement «des comparatives méta-énonciatives » (AuthierRevuz, 1995: 115) et peuvent être considérées, au même titre que les propositions introduisant la source 
d'un discours rapporté (comme l'a affirmé hier le premier ministre...) comme des adjoints parenthétiques (Desmets et Roussarie, 2001). Nous allons examiner successivement ces deux types.

\subsubsection{Les structures en comme ou adjoints parenthétiques}

Authier-Revuz, 1995 (115) et Debaisieux et Martin (2010 : 321) ont signalé le fait que les structures en comme introduisent majoritairement «des comparatives méta-énonciatives» ${ }^{3}$. La construction en comme avec verbe au passé composé à valeur d'accompli, ou un futur simple ou périphrastique est très productive dans l'écrit scientifique : comme nous avons pu le voir (le constater, l'observer etc.), comme nous allons le voir, on le verra ... etc. La caractéristique de ces constructions est souvent principalement une fonction métatextuelle et/ou évidentielle : l'auteur signale, que le fait dont il rend compte a déjà été constaté (étayé, prouvé...) ou va l'être; la structure joue alors essentiellement un rôle rhétorique de renforcement de l'argumentation et/ou de guidage du lecteur dans le plan de texte. On peut cependant distinguer clairement deux sous-catégories de routines en comme : la première comporte un localisateur (ex. comme nous l'avons vu dans notre première partie ; comme nous le voyons sur la figure 10, ...). Dans ce premier cas, la fonction de rappel ou de co-constat est privilégiée ; dans le second, il n'y a pas de localisateur, et la routine est très proche, du point de vue de son fonctionnement des routines avec incise progressive.

\subsubsection{Les routines avec incise progressive}

Rappelons certaines caractéristiques de voir en incise (on l'a vu). On peut remarquer en effet que cette construction, à valeur métadiscursive permettant la prise à témoin du lecteur, n'est pratiquement pas mobilisée avec les autres VOC (observer, constater, remarquer, noter, etc.). Dans les genres didactiques ou argumentatifs - dans lesquels on peut inclure les genres scientifiques - l'utilisation de routines rhétoriques telles que on l'a $v u$, permet d'associer le lecteur à l'argumentation. Ce type d'incises comportant une anaphore pronominale - ainsi que les formes correspondantes en comme sans localisateur (comme on l'a vu), sont des signaux de présupposition qui ne supportent pas la négation. Les exemples suivants illustrent bien ce phénomène :

7) ? X, nous ne l'avons pas vu, est similaire à $Y$.

8) ? X, nous ne l'avons pas vu sur la figure $Z$, est similaire à $Y$.

On l'a vu, routine à incise progressive, et comme on l'a vu, adjoint parenthétique utilisé sans localisateur fonctionnent de manière très proche, si bien qu'il est naturel de les considérer comme deux variantes d'une même construction (au sens des grammaires de construction). Il s'agit de deux structures fortement pragmaticalisées, et assez figées, au moins dans l'écrit scientifique; en effet, si en théorie, on pourrait trouver d'autres choix lexicaux et d'autres tiroirs verbaux ([comme] on l'aura noté, [comme] on peut le remarquer...), ce type d'exemples reste extrêmement rare dans le corpus. Cette spécificité s'explique également par le caractère propre du verbe voir, sur lesquelles nous revenons plus loin.

\subsubsection{Les routines à complétive ou à SN objet}

Nous regroupons ici les formes en complétive et celles en SN objet dans la mesure où elles jouent fondamentalement le même rôle dans l'écrit scientifique. Rappelons notre hypothèse initiale, selon laquelle ces structures ont pour fonction centrale l'exposition proprement dite des résultats; il s'agit de constructions avec nous ou on : on se rencontre plus souvent dans le co-constat, et la structure constative $\mathrm{y}$ endosse en outre une fonction dialogique. Le présent de l'indicatif, à la forme active, est largement privilégié dans ces emplois ${ }^{4}$. La complétive conjonctive - la plus fréquente dans le corpus (nous voyons que la température remonte) représente la structure prototypique, celle avec complément nominal intégrant souvent un déverbal (nous constatons une légère remontée de la température). Il existe cependant des préférences de construction suivant les verbes : ainsi observer est le seul verbe à privilégier - et de manière assez nette - une construction avec un objet nominal (277 occurrences de SN contre 134 
QUE_P). Les objets nominaux peuvent être des noms de processus (dilution, augmentation), ou marquer un résultat (tendances, disparités) :

9) ? Nous observons une déstabilisation énergétique de 25 Kcal.

Les QUE_P autorisent cependant des constats du type généralisant, ce qui ne peut être obtenu par un SN :

10) En ce qui concerne le clip Hotdog, on observe que tous les énoncés contiennent des adverbiaux temporels ; [lin-com-69-body]

La structure complétive permet donc à la fois le constat lié à la mise à jour d'un procès (ce que permet aussi la structure avec $\mathrm{SN}$ ) et le constat généralisant.

Dans l'emploi avec complétive, le verbe observer - de même que voir - est fréquemment utilisé avec un locatif, en raison de sa motivation visuelle :

11) On observe sur la Figure 58 que les activités de réparation... [bio-the-95-body]

12) Dans un noyau inter-phasique, on peut observer par microscopie optique deux types de structures chromatiniennes [bio-the-281-introduction]

Ces deux types de structures sont donc étroitement associés au système de la preuve dans les écrits scientifiques.

\subsection{Les cas des verbes /voir / et /s'apercevoir /}

Le verbe voir mérite d'être étudié à part, en raison du fait qu'il est mobilisé dans des routines phraséologiques très variées. Utilisé au présent, avec on et nous, avec et sans le modal pouvoir, il se caractérise par une fréquence assez élevée (il arrive en troisième position, avec 333 occ.) mais surtout par la diversité de ses emplois et de ses constructions. Dans le corpus Scientext, l'emploi avec l'indéfini on domine très largement, et le modal reste assez peu fréquent. En dehors des structures avec comme et ou sans comme, du type on le voit déjà mentionnées, il introduit le plus souvent une proposition complétive conjonctive, moyen prototypique de signaler un constat que l'on veut faire partager au lecteur. Mais on trouve également avec voir :

- des interrogatives indirectes, utilisées notamment pour marquer le haut degré (on voit à quel point, on voit combien... ) ;

- des propositions infinitives, recourant souvent à des verbes à valeur aspectuelle inchoative, du type apparaître, émerger, poindre, se dessiner, surgir, se profiler :

13) Mais parallèlement, nous voyons une seconde tendance émerger entre N3 et N5, que nous appellerons morphographique.

Certaines d'entre elles peuvent revêtir une valeur évaluative (en particulier avec on voit bien), en fonction du nom, sujet de la proposition infinitive :

14) On voit bien ressortir ici les présupposés évidents à la conception...

La construction infinitive, après un verbe de perception, est souvent décrite comme favorisant une interprétation moins cognitive que la construction complétive ; en (13) le sens perceptuel n'est certes guère présent, mais la construction infinitive tend à mettre en valeur l'acte même du constat ; en (14), avec l'adverbe bien, on trouve un emploi proche de celui qui est analysé plus bas, à propos des SN à valeur évaluative, et donc une interprétation cognitive.

Les extractions en tête de phrase jouent un rôle de «pont » anaphorique ou cataphorique, en introduisant un exemple (ou une autre donnée) permettant d'illustrer, ou de confirmer ce qui vient d'être dit :

15) C'est ce que l'on voit dans l'exemple suivant... 
Enfin, on rencontre un nombre non négligeable de SN en fonction objet (84 occ. sur 240). Dans ce type d'emplois, le SN est généralement abstrait et peut avoir une valeur évaluative :

16) On voit ici la complexité, la difficulté, l'intérêt, les limites (de l'analyse, de cette question, de la méthode utilisée...).

Ce que l'on «voit» n'est donc pas toujours un résultat, mais parfois un élément permettant une considération sur la recherche elle-même, avec par conséquent une valeur métatextuelle. On peut également trouver d'autres types d'objets, par exemple lorsqu'il s'agit de synthétiser des résultats : dans l'exemple (17), l'utilisation conjointe d'observer traduit bien la complémentarité des deux verbes :

17) Toutefois, si on observe les adjonctions de marques plurielles à l'infinitif, on voit trois tendances, valides à tous les niveaux scolaires... [lin-hdr-396-body]

Avec le clitique $\boldsymbol{y}$ anaphorique, la structure avec voir permet de fournir une interprétation issue des données précédemment présentées :

18) On peut y voir l'effet de certaines pratiques idiomatiques dans la mesure où... [lin-the-27body]

De la même façon, la structure avec le clitique en est utilisée pour ajouter un exemple illustrant ce qui vient d'être dit :

19) On en voit en particulier un exemple avec les circonstancielles «si ... alors » à valeur générique [lin-the-27-body]

Enfin, on note aussi, plus rarement, l'emploi de participes passés à valeur passive :

20) On voit donc clairement illustré ici le principe d'équivalence fonctionnelle) ;

Les constructions avec infinitives et avec participes passés à valeur passive sont spécifiques du verbe voir et on ne les retrouve pas avec les autres verbes. Ce n'est évidemment pas le cas des conjonctives, très nombreuses quel que soit le verbe de constat. A quelles fins sont utilisées les conjonctives après voir ? Deux types d'emplois se détachent dans le corpus :

- le constat conclusif (après un développement argumentatif), à fonction interprétative :

21) On voit donc que, tout bien pesé, l'orthographe du français ne représente pas un cas très satisfaisant d'optimalité sémiographique. [lin-art-99-body]

Le constat conclusif, issu du développement argumentatif qui précède, n'a pas obligatoirement besoin de prendre appui sur une localisation précise dans le texte. Il est assez fréquent cependant qu'un adverbial de lieu soit mobilisé (on voit ici, on voit par là que...). Cet adverbial ayant valeur anaphorique semble se rapprocher cet emploi du fonctionnement des structures en comme. Cependant, il s'agit bien d'un constat au sens fort du terme, puisqu'il est effectué à partir des éléments qui précèdent, et non simplement rappelé, comme dans le fonctionnement de voir en incise. L'utilisation du on, nettement privilégiée dans le constat conclusif, marque la volonté d'associer le lecteur à la conclusion qui est tirée ;

- le constat d'observation partagée ou co-constat : il repose sur l'appel à l'observation d'une figure, d'un graphique ou d'un exemple ou de toute autre donnée comme appui à la démonstration (généralement indiquée par un déictique (ici, là...) ou un syntagme prépositionnel), le lecteur étant invité à s'y reporter :

22) On voit clairement sur ce graphique que la seule présence d'une bouche souriante quelle que soit l'expression dans laquelle cette bouche est insérée, correspond à une augmentation nette du pourcentage de reconnaissance. [psy-com-112-body]

La construction $[\mathrm{ON}+$ voit + Adv $(-$ ment $)+$ prép. + LOC. + Que_P ] qu'illustre le dernier exemple est particulièrement productive dans le corpus. Rappelons que dans le cas du constat d'observation partagée, on trouve fréquemment un localisateur permettant de renvoyer à un élément péri-textuel (figure, 
graphique) ou à une portion du texte, sémiographiquement identifiable (exemple numéroté, paragraphe ou partie du texte, etc.).

En dehors de ces deux emplois principaux, voir permet aussi des évaluations ou des commentaires, voire des précisions, comme cet emploi avec ici, fourni en note :

23) On voit ici que le modèle de la mémoire demanderait à être précisé : les représentations d'individus ou catégories évoquées par les symboles ne devraient pas avoir le même statut que celles effectivement perçues. [tal-the-641-notes]

On sort ici du constat proprement dit, pour anticiper les objections possibles du lecteur, dans le cadre de la stratégie argumentative, on voit que marquant aussi la connivence avec le lecteur, en présupposant son adhésion à ce qui est dit. La plasticité de voir, la diversité même des constructions dans lesquelles il entre en font un outil à (presque) tout faire : co-constat, constat conclusif, marqueur évaluatif (on voit bien...), marqueur d'inférence, marqueur de présupposition.

Disons également un mot du verbe s'apercevoir: ce verbe a une fréquence faible dans le corpus (23 occurrences dont 20 avec l'indéfini : on s'aperçoit). La forme modalisée est totalement absente. Le quasi synonyme se rendre compte, d'un emploi très proche, comporte quant à lui une dizaine d'occurrences dans le corpus. La structure utilisée est presque exclusivement celle en QUE_P (1 seule occurrence avec $+\mathrm{SN})$. Le verbe $s$ 'apercevoir est utilisé pour marquer le fait qu'un fait se révèle au chercheur à l'issue du processus d'observation. Il est donc toujours perfectif contrairement à voir, qui peut être perfectif ou imperfectif. C'est ce qui explique que, comme nous l'avions déjà noté à propos de la relation voir / observer, l'utilisation de s'apercevoir s'effectue souvent concomitamment avec, dans le cotexte qui précède, un verbe d'observation tel que considérer (en 24) ou observer (en 25).

24) Si l'on considère des acides aminés individuels, seuls ou dans de très petits peptides, on s'aperçoit que chacun peut occuper de l'ordre de dix conformations. [bio-art-184introduction]

25) En observant de plus près ces productions, on s'aperçoit qu'une consonne prédomine : la consonne $/ \mathrm{n} /$. [lin-the-23-body]

Le verbe s'apercevoir fonctionne mal avec un circonstant de lieu :

26) ? On s'aperçoit sur la Figure 58 que les activités de réparation (....). [bio-the-95-body, modifié]

En revanche, comme en témoignent les exemples (24) et (25), il s'adapte parfaitement aux structures hypothétiques, ou au gérondif, qui permet de marquer le procès à l'issue duquel le constat s'impose. 


\section{Conclusion}

Il est temps de faire le point sur les différents types de routines observées dans le corpus. Une typologie de ces formes peut s'effectuer selon plusieurs logiques, que nous avons croisées au cours de l'étude. Une première approche consiste à partir de la structure syntaxique. Dans ce cas, on identifie très clairement deux « familles » de routines phraséologiques liées aux formes du constat :

- d'une part, les routines correspondant à des structures parenthétiques, au sein desquelles il est possible de distinguer :

a) les incises progressives - n'ayant pas d'autonomie sémantique et syntaxique - que l'on rencontre exclusivement dans le corpus avec un anaphorique (ex. on le voit);

b) les structures en comme, qui peuvent être analysées comme des adjoints parenthétiques.

- d'autre part, les routines dans lesquelles le verbe est suivi d'un objet, qu'il s'agisse d'une complétive ou d'un SN (on constate que ... on constate une diminution ...).

Sur le plan fonctionnel, cette première approche permet d'opposer les deux principales formes liées à l'observation et au constat dans l'écrit scientifique. La première famille correspond à un constat incident, ou notation d'arrière-plan (lié à la prédication seconde), l'objectif étant alors essentiellement pour le scripteur de renforcer son argumentation en rappelant ce sur quoi s'appuie sa recherche ou de le renvoyer à d'autres parties du texte. La seconde famille correspond au constat scientifique proprement dit, l'enjeu étant alors de produire au lecteur, de manière visible, les observables sur lesquels s'appuie l'assertion constative.

Cependant, cette présentation apparaît un peu trop simple à l'examen. En effet, nous avons pu distinguer deux types de structures en comme : les unes, sans localisateur, et très figées dans leur emploi (comme on le voit, comme on peut le constater) fonctionnent effectivement comme de simples béquilles visant à renforcer l'argumentation. Les autres, comportant un localisateur (comme on peut le voir sur la figure 10), sont liées à la co-observation et jouent un rôle central dans le processus d'administration de la preuve.

Il est donc nécessaire de compléter l'entrée syntaxique de départ par une description plus fine des rôles sémantiques et énonciatifs impliqués en décrivant les constructions. Celles-ci doivent prendre en compte des informations sur l'énonciateur (nous ou on, on étant plus fréquemment associés au co-constat), des informations sur la présence ou non d'un localisateur. La sémantique des verbes joue également un rôle central : un verbe comme voir a une très grande plasticité, puisqu'il peut être utilisé aussi bien comme quasi verbe de perception, dans le co-constat (l'auteur scientifique demande au lecteur pair d'examiner des données) que pour montrer l'issue d'un raisonnement inférentiel (nous voyons que...), à l'issue d'un processus d'observation. Enfin, la dimension textuelle ne peut être ignorée, le constat pouvant être conclusif ou interprétatif, à l'issue d'un développement, ou bien se limiter à un simple relevé de données, lors de la phase d'analyse. Les notions d'observation et de constat, si elles trouvent facilement une traduction linguistique, sont donc elles-mêmes polymorphes et correspondent à des activités assez diverses au plan cognitif tout comme au plan de l'interaction sociale.

Il est cependant possible, à partir de l'identification de constructions unissant formes et sens, de repérer les routines les plus saillantes, parce qu'elles se sont figées dans l'usage. A l'issue de notre examen, il apparaît que les parenthétiques progressives et les structures en comme sont de bonnes candidates à cet emploi routinier ; les structures en comme sans localisateur jouent un rôle essentiellement rhétorique et argumentatif; les structures avec localisateur n'ont pas le même caractère routinier; elles mettent cependant en place un système extrêmement stable et clair, avec des constructions productives, comme nous l'avons vu dans l'exemple de la construction [ON + voit + Adv (-ment) + prép. + LOC. + Que_P ]. Les phraséologismes étudiés sont donc de deux ordres: certains sont des formes en voie de pragmaticalisation, tandis que les autres sont plutôt des constructions, i.e. des moules productifs, qui contraignent fortement les substitutions paradigmatiques. Notre étude confirme également l'importance 
de la dimension générique pour l'étude des phraséologismes, même lorsqu'ils ne sont pas propres à un champ disciplinaire : qu'il s'agisse des formules pragmaticalisées ou des constructions, on a affaire, en ce qui concerne les formes du constat scientifique, à des formes assez spécifiques ; certaines d'entre elles cependant (notamment l'incise progressive on l'a $v u$ ) se rencontrent plus généralement dans les genres didactiques. Il serait certainement intéressant d'élargir cette étude des formes du constat à d'autres genres : le constat policier ou juridique (par ex. le constat d'huissier) ou la fiction romanesque (par ex. le roman policier).

\section{Références bibliographiques}

Andersen, H.L. (1997). Propositions parenthétiques et subordination en français parlé. Université de Copenhague. [Thèse de doctorat]

Apothéloz, D. (2002). « La rection dite 'faible' : grammaticalisation ou différentiel de grammaticité ? ». Verbum, 25/3, 241-262.

Authier-Revuz, J. (1995). Ces mots qui ne vont pas de soi. Boucles réflexives et non-coïncidences du dire. Paris : Larousse.

Beyssade, Cl. (2012). Le statut sémantique des incises et des incidentes en français. Langages, 186, 115-130.

Borillo, A. (1982). Deux aspects de la modalité assertive : croire et savoir. Langages, 67, 33-53.

Cappeau, P. et Savelli, M. (2001). C'est bien comme ça ? Etude des constructions en comme. Recherches sur le français parlé, 16, 39-62.

Cornulier, B. de (1978). L'incise, la classe des verbes parenthétiques et le signe mimique. Cahiers de linguistique, 8, 53-95.

Debaisieux, J.-M. \& Martin, Ph. (2010). Les parenthèses, étude macrosyntaxique et prosodique sur corpus. M.-J. Béguelin, G. Corminboeuf \& M. Avanzi (éd.). La parataxe, tome 2, Structures, marquages et exploitations discursives, Berne, Peter Lang, 307-338.

Delplanque, A. (2006). Juger d'après les apparences : le cas du français. Corela, Les verbes d'apparence. Publié en ligne le 13 mars 2006. URL : http://corela.edel.univ-poitiers.fr/index.php?id=1284.

Desmets, M. \& Roussarie, L. (2000). French reportive comme clauses: A case of parenthetical adjunction. In Flickinger, D. et Kathol, A. (éd.), Proceedings of the 7th International HPSG Conference : UC Berkeley, 43-62.

Fløttum, K. (2004). La présence de l'auteur dans les articles scientifiques : étude des pronoms je, nous et on. In Auchlin, A. Burger, M. Filliettaz, L. \& Grobet A. (éd.) Structures et discours, Mélanges offerts à Eddy Roulet, Québec : Nota Bene, 401-416.

Fløttum, K., Dahl, T. \& Kinn, T. (2006). (éd.) Academic Voices across languages and disciplines. Amsterdam/Philadelphia, John Benjamins.

Fløttum, K., Jonasson, K. \& Noren, C. 2007. (éd.) On, pronom à facettes. Louvain, Duculot.

Fuchs, C. \& Le Goffic, P. (2005). La polysémie de comme. In Soutet, O. (éd.), La polysémie, Paris, Presses de l’Université Paris Sorbonne, 267-291.

Gachet, F. \& Avanzi, M. (2011). Description prosodique des « recteurs faibles en incise ». In Yoo, H.-Y., \& DelaisRoussarie, E. : Actes d'IDP 2009, septembre 2009: Paris, 173-189. URL: $\mathrm{http} / /$ makino.linguist.jussieu.fr/idp09/docs/IDP_actes/Articles/gachet.pdf

Grossmann, F. (2013). Les verbes de constat dans l'écrit scientifique. In Tutin, A. et Grossmann, F. (éd.) L'écrit scientifique, Du lexique au discours, Rennes, Presses Universitaires de Rennes, 85-100.

Grossmann, F. (2014). Verbes de constat et autres verbes «parenthétiques ». Quel statut dans l'écrit scientifique ? Arena Romanistica, 14, 106-122. 
Grossmann F., Tutin A. (2010a). Evidential Markers in French Scientific Writing: the Case of the French Verb 'voir'. In Smirnova E., Diewald G. (éd.): Evidentiality European Languages, Empirical Approaches to Language Typology (EALT). Berlin, New York: Mouton de Gruyter, 279-307.

Grossmann F., Tutin A. (2010b). Les marqueurs verbaux de constat : un lieu de dialogisme dans l'écrit scientifique. Actes du colloque Dialogisme : langue, discours 2010, 8-10 septembre 2010. Montpellier, Praxiling. Disponible à l'adresse : http://www.univ-montp3.fr/praxiling/IMG/pdf_GrossmannTutin.pdf

Nølke, H. (1994). La dilution linguistique des responsabilités. Essai de description polyphonique des marqueurs évidentiels 'il semble que' et 'il parait que'. Langue française, 102, 84-94.

Recanati, F. (1984). Remarques sur les verbes parenthétiques. In Attal, P. et Muller, Cl. (éd.) : Actes du Colloque de Rennes, De la syntaxe à la pragmatique, Université de Haute-Bretagne: Lingvisticae Investigationes Supplementa, vol.8, 319-353.

Tuchais, S. (2012). Les verbes d'opinion et la modalisation en français: état des lieux et perspectives. Bulletin of the Faculty of Foreign Studies, Sophia University, 47, 153-172.

Tutin, A. (2010). Dans cet article, nous souhaitons montrer que..., Lexique verbal et positionnement de l'auteur dans les articles de Sciences Humaines. Lidil, 41, 15-40.

Tutin, A., \& Grossmann, F. (2013). L'écrit scientifique : du lexique au discours. Autour de Scientext. Rennes, Presses Universitaires de Rennes.

Urmson, J. O. (1952). Parenthetical Verbs. Mind, 61, 480-496.

Wilmet, M. (1998, $2^{\mathrm{e}}$ éd.). Grammaire critique du français. Paris, Bruxelles, Hachette et Duculot.

\footnotetext{
${ }^{1}$ Nous regroupons observation et constat, en raison de la très forte proximité tant sémantique (cf. observer que et constater que) qu'épistémique : l'observation précède généralement le constat proprement dit, mais se confond aussi parfois avec lui.

${ }^{2}$ Pour une présentation plus détaillée, voir Grossmann, 2013.

${ }^{3}$ Ce point est également signalé par Cappeau \& Savelli (2001)

${ }^{4} \mathrm{D}$ 'autres formes peuvent mériter attention, en particulier des formes à valeur jussive telles que il est à noter que, il faut noter que ou l'impératif de première personne : constatons que.
} 